\title{
Review of: "Reconstruction of sound driven, actively amplified and spontaneous motions within the tree cricket auditory organ"
}

Ben Warren ${ }^{1}$

1 University of Leicester

Potential competing interests: The author(s) declared that no potential competing interests exist.

The reviewer(s) wrote their comments in the PDF attached in the Supplementary data section of the present review. 